\title{
Cervicothoracic arachnoiditis-a rare complication of aneurysmal intracranial subarachnoid haemorrhage
}

\author{
Shyam S. Swarna $\mathbb{1}^{1} \cdot$ David McKean $^{2} \cdot$ Maurizio Belci $^{3}$
}

Received: 14 April 2018 / Revised: 30 May 2018 / Accepted: 30 May 2018

(c) International Spinal Cord Society 2018

\begin{abstract}
Introduction Subarachnoid haemorrhage is a catastrophic condition which has significant morbidity and mortality, with mortality rate ranging between $8-67 \%$ and is associated with significant morbidity among the survivors. Arachnoiditis of the spinal cord is a very rare complication which can occur months to years after the subarachnoid haemorrhage.

Case presentation We report a case of cervicothoracic arachnoiditis following the subarachnoid haemorrhage secondary to posterior inferior cerebellar artery (PICA) aneurysm with posterior fossa AVM (arterio-venous malformation) causing dense paraplegia.

Discussion The haemorrhage that occurs into the fourth ventricle passes to the subarachnoid space via foramen of Magendie and Luschka. This subsequently induces inflammatory changes of the meninges predisposing to arachnoiditis. Early recognition and intervention may prevent this devastating complication or at least reduce the morbidity.
\end{abstract}

\section{Introduction}

Subarachnoid haemorrhage is a devastating condition with high incidence of mortality rate and those who survive have a greater incidence of morbidity [1-4]. Though rare, arachnoiditis of the spinal cord has been previously reported in literature [5]. We present a 55-year-old gentleman who following aneurysmal intra cranial subarachnoid haemorrhage developed bilateral leg pain within the next few days which was not investigated as he had pre-existing Sciatica. This gentleman went on to develop complete paraplegia within 12 weeks of the initial presentation. In spite of an attempt at adhesiolysis he showed no improvement and had established irreversible neurological deficit. Hence, we report this case to create awareness that though the arachnoiditis is a rare complication, there should be a high

Shyam S. Swarna

shyamswarna@yahoo.co.in

1 Rehabilitation Medicine, Oxford University Hospitals NHS Trust, Oxford, UK

2 Radiology Department, Stoke Mandeville Hospital, Buckinghamshire Healthcare NHS Trust, Aylesbury, UK

3 National Spinal Injuries Centre, Stoke Mandeville Hospital, Buckinghamshire Healthcare NHS Trust, Aylesbury, UK index of suspicion in patients with lower limb symptoms following intracranial subarachnoid haemorrhage. Early intervention might salvage the devastating complication if this is diagnosed at very early stages.

\section{Case presentation}

Fifty-five-year-old previously fit and healthy gentleman presented to an acute hospital with collapse and sudden onset of headache. CT head showed subarachnoid haemorrhage with hydrocephalus. CT angiogram showed posterior inferior cerebellar artery (PICA) aneurysm with posterior fossa arterio-venous malformation (Fig. 1). The following morning, he underwent clipping of the aneurysm. He made good progress post operatively. During the following days, he started complaining of bilateral leg pain. Since he had background history of long-standing sciatica, he was discharged with regular analgesia.

Ten weeks later, he presented to the acute hospital with sudden onset chest pain. He underwent routine investigations to rule out myocardial ischaemia, pulmonary embolism, and chest infection, and was discharged home. A week after the discharge he noticed numbness below the chest with difficulty in walking. He started showing signs of urinary incontinence. MRI scan of the spine showed spinal arachnoiditis (Fig. 2a, b) due to meningeal irritation 


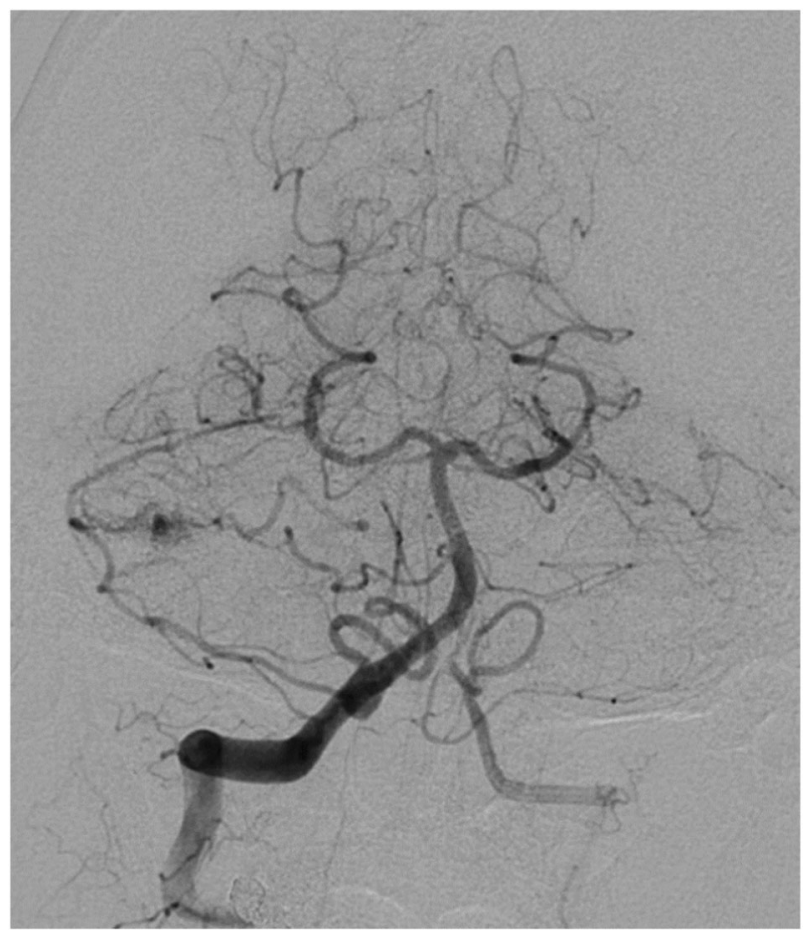

Fig. 1 Preoperative digital subtraction angiogram (DSA) images in the arterial phase. The DSA reveals the presence of an aneurysm arising from the right posterior inferior cerebellar artery with no evidence of cerebral vasospasm

secondary to subarachnoid haemorrhage. He showed progressive weakness of both lower limbs with bladder symptoms suggestive of neurogenic bladder.

He was transferred to a tertiary centre for neuro-surgical intervention. He underwent urgent laminectomy. It was noted that the spinal cord was pale and pulseless. An attempt to place a lumbar drain was futile and the surrounding adhesions could not be removed (Fig. 3). Post operatively he was treated initially with intravenous Methyl prednisolone and changed to oral steroids which was tapered down in 2-3 weeks' time.

He was later transferred to the spinal rehabilitation centre for ongoing rehabilitation. He was found to have dense paraplegia with sensory deficit. His neurological level was T6 AIS A. His bladder remained catheterised and later went on to have supra pubic catheter. His bowel management required regular suppositories. He continues to have neuropathic pain involving both lower limbs which is being managed with tricyclic antidepressants. He has remained wheelchair dependent for mobilisation.

\section{Discussion}

Spinal arachnoiditis was first reported by Nelson in 1943 [6] in a patient with previous history of spinal haemorrhage

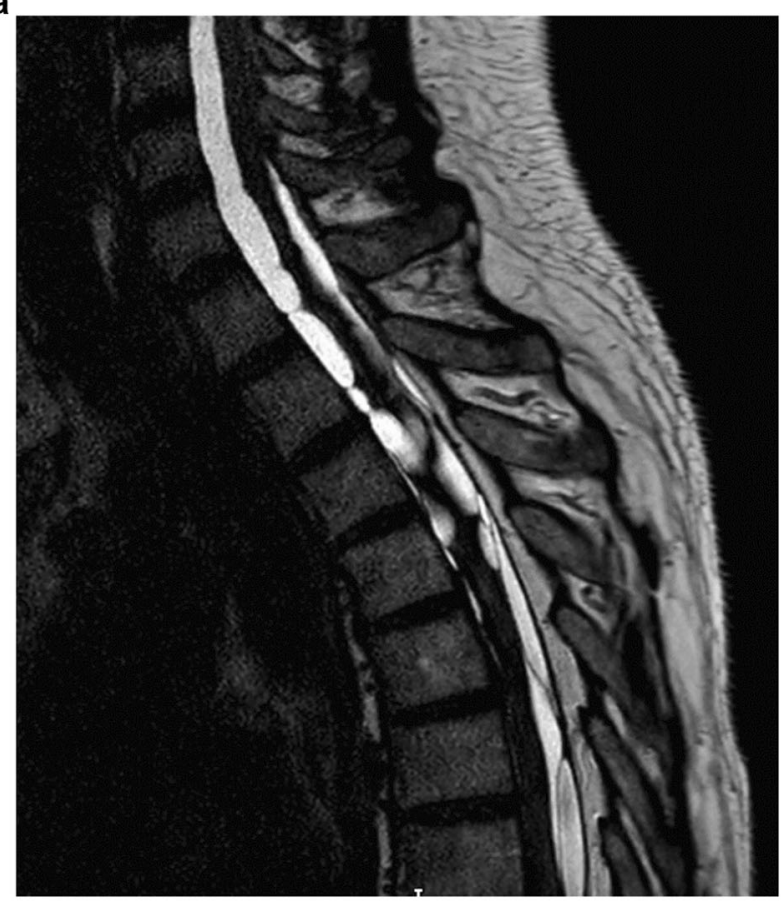

b

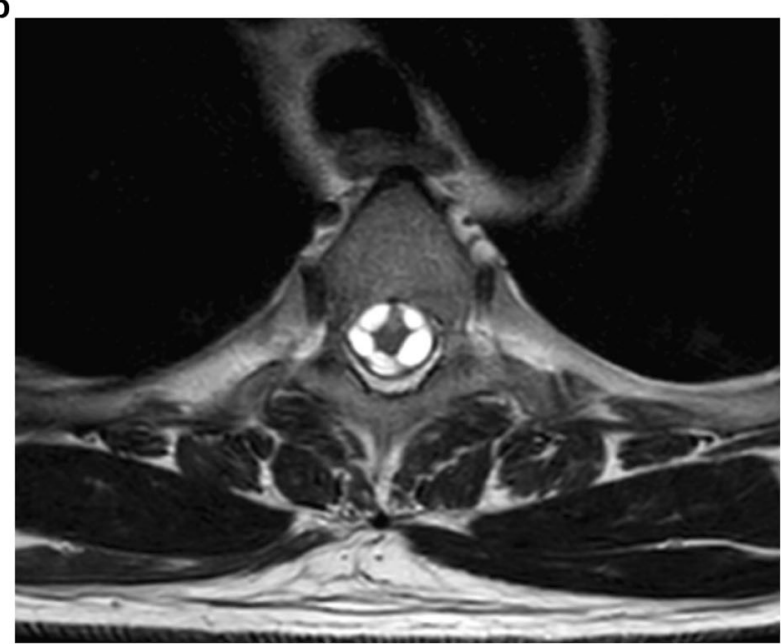

Fig. 2 a Preoperative T2-weighted DRIVE sagittal image of the cervicothoracic spine revealing multiple dural adhesions distorting and compressing the spinal cord with loculated dorsal cysts. b Axial T2weighted image of the thoracic cord revealing multiple fibrous bands tethering and distorting the cord

secondary to thrombocytopaenic purpura. The patients reported in various case reports were in the age group twenties to late sixties (22-69 years) [7] and the previous case reports showed female preponderance $(86 \%)$.

Spinal arachnoiditis presentation is secondary to the level of involvement and degree of compression. Usual presentation is leg pain, progressive weakness, sensory deficits with sphincter dysfunctions. Twenty-five cases were evaluated in the previous case report [7], of which 13 patients had primary subarachnoid haemorrhage secondary to PICA 


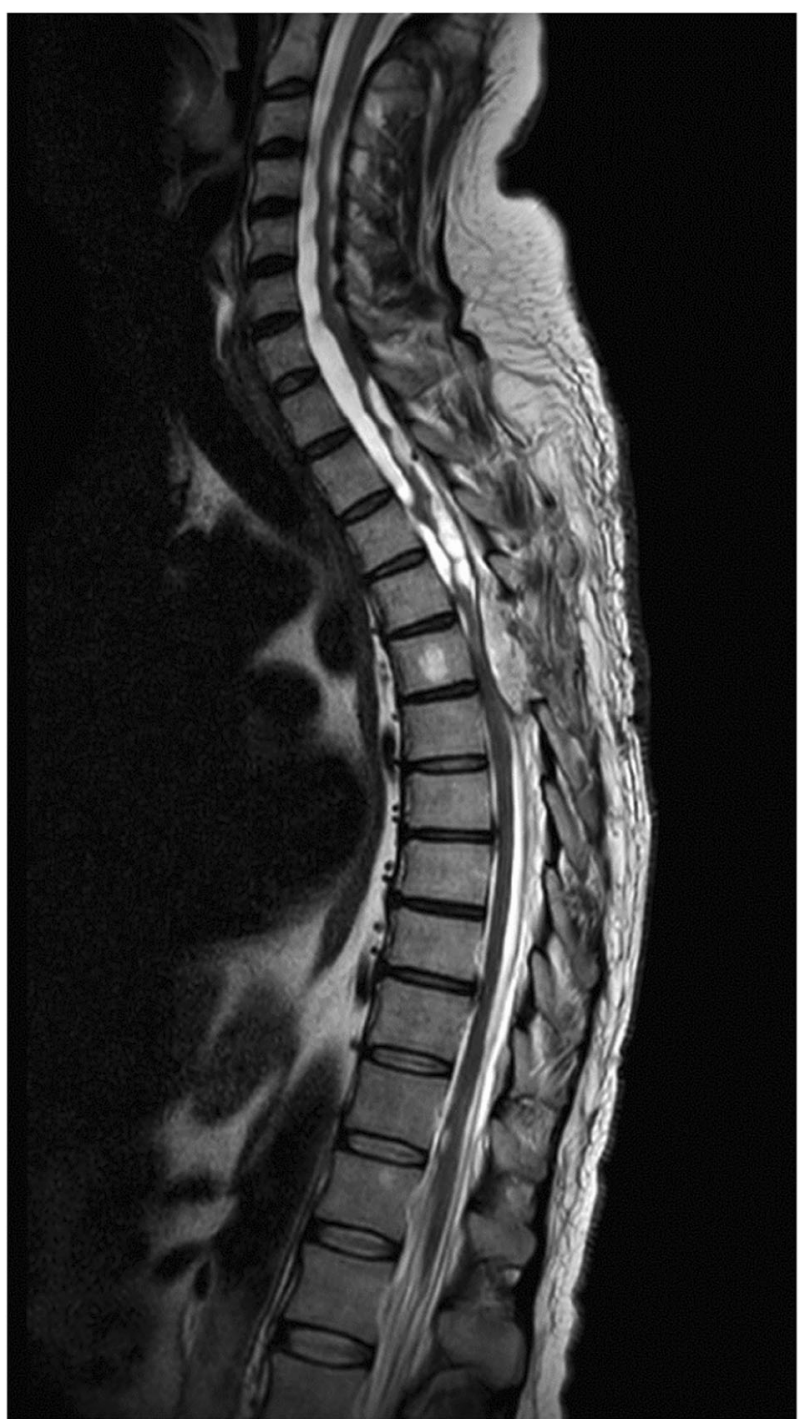

Fig. 3 Postoperative sagittal T2-weighted images demonstrating persistent dural adhesions distorting the spinal cord

aneurysm, two with posterior communicating artery aneurysm, and only one patient had aneurysm involving the anterior communicating artery [8]. Three patients presented with progressive paraparesis with urinary incontinence, five patients with progressive paraparesis with sensory loss. Twelve patients with only paraparesis, one patient with Cauda equina syndrome [9], and three patients with illdefined symptoms [10] and one patient with sciatic pain and urinary urgency.

Most of the cases reported showed spinal arachnoiditis involving thoracic area [7], though there is one reported involving cervical vertebra [11] and three involving the lumbar area $[12,13]$. The duration between the subarachnoid haemorrhage and arachnoiditis ranged between 30 days to 12 years.

The hypothesis is that haemorrhage occurs in the fourth ventricle of posterior fossa, peri mesencephalic area, and cerebellopontine cisterna magna [7]. The blood that accumulates in these regions passes to the subarachnoid space via foramina of Magendie and Luschka [5]. Hydrocephalus is a common complication following subarachnoid haemorrhage due to blocked cerebro-spinal fluid circulation [7].

It is understood from the previous reports that subarachnoidits secondary to subarachnoid haemorrhage is due to blockage of basal cisterns by the blood [14]. The accumulation of blood products and further haemolysis [15] in the subarachnoid space leads to irritation of meninges and eventually arachnoiditis. Blood products in the initial stages initiates inflammatory response by stimulating massive influx of inflammatory cells with release of cytokines [16]. This potentiates the fibroproliferation anywhere in the neuro-axis which leads to collagen synthesis. The fibrous tissue formation along the neuro-axis leads to obliteration of the subarachnoid space, adhesion, tethering around the spinal cord. One case [17] was reported to have spinal migration of subarachnoid haemorrhage due to ruptured vertebral artery aneurysm. The other hypothesis is vasospasm of either anterior and posterior spinal arteries or from radicular arteries due to haemorrhage in the subarachnoid space [18].

Syringomyelia is a complication in a very small subset of patients with spinal arachnoiditis following aneurysmal subarachnoid haemorrhage $[4,8,14,15]$.

The shunting of cerebro-spinal fluid or subarachnoid space reconstruction [19-22] are well-recognised procedures for synrigomyelia due to adhesive arachnoiditis. Microsurgical arachnoidolysis [23], a technically demanding procedure, has been reported to mobilise spinal cord and to reconstruct CSF pathways for localised arachnoiditis with favourable outcome. Duraplasty using fascia lata [24] graft has been reported as an established procedure to maintain CSF pathways followed by expansive laminoplasty [25, 26]. Three out of four patients reported by Hiroki Morisako et al. [24] showed moderate improvement in the neurological function following microsurgical arachnoidolysis. Subarachnoid-subarachnoid bypass [27] has been reported as a surgical option to bypass localised adhesive arachnoiditis with arachnoid cyst which has shown improvement in the neurological status. Thecaloscopy (Flexible endoscopy) [28] is considered as a surgical treatment for spinal arachnoiditis even in the diffuse forms with no serious complications.

The prognosis of this condition is entirely based on the degree of adhesions and location of the pathology.

\section{Conclusion}

Spinal arachnoiditis though a very rare complication of intracranial subarachnoid haemorrhage can lead to non- 
reversible morbidity. Hence, a high index of suspicion is required to identify early symptoms like radicular pain, paraesthesia, weakness, or bladder and bowel symptoms when assessing patients following intracranial subarachnoid haemorrhage.

Acknowledgements The authors would like to thank the patient who agreed to have his case reported. We also acknowledge the National Spinal Injuries Centre, Stoke Mandeville, UK, for letting us use the resources towards this manuscript.

\section{Compliance with ethical standards}

Conflict of interest The authors declare that they have no conflict of interest.

\section{References}

1. Rivero Rodriguez D, et al. Predictor's of mortality in patients with aneurysmal subarachnoid haemorrhage and re-bleeding. Neurol Res Int. 2015;2015:545407.

2. Augustijn P, Vanneste J, Davies G. Chronic spinal arachnoiditis following intracranial subarachnoid haemorrhage. Clin Neurol Neurosurg. 1989;91:347-50.

3. KoK AJ, Verhagen WI, Bartels RH, Van Dijik R, Prick MJ. Spinal arachnoiditis following subarachnoid haemorrhage. Report of two cases and review of the literature. Acta Neurochir. 2000;142:795-8

4. Abel TJ, Howard MA, Menezes A. Syringomyelia and spinal arachnoiditis resulting from aneurysmal subarachnoid haemorrhage: report of two cases and review of the literature. J Craniovertebral Junction Spine. 2014;5:47-51.

5. Gupta S, Soellinger M, Boesiger P, Poulikakos D, Kurtcuoglu V. Three-dimensional computational modelling of subject-specific cerebrospinal fluid flow in the subarachnoid space. J Biomech Eng. 2009;131:021010.

6. Nelson J. Intramedullary cavitation resulting from adhesive spinal arachnoiditis. Arch Neurol Psychiatry. 1943;50:1-7.

7. Basaran R, Kaksi M, Efendioglu M, Onoz M, Balkuv E, Kaner T. Spinal arachnoid cyst associated with arachnoidits following subarachnoid haemorrhage in adult patient: a case report and literature review. Br J Neurosurg. 2015;29:285-9.

8. Eneling J, Bostrom S, Rossitti S. Subarachnoid haemorrhage associated arachnoiditis and syringo myelia. Clin Neuroradiol. 2012;22:169-73

9. Whetstone KE, Crane DA. Cauda equina syndrome resulting from lumbar arachnoiditis after intracranial subarachnoid haemorrhage: a case report. PMR. 2013;5:539-41.

10. Lombardi G, Passerini A, Migliavacca F. Spinal arachnoiditis. Brit J Radiol. 1962;35:14-20.
11. TJandra JJ, Varma TRK, Weeks RDW. Spinal arachnoiditis following subarachnoid haemorrhage. Aust NZJ Surg. 1989;59:84-7.

12. Lorenzana-Honrado L, Cabezudo-Artero JM, Gozez-Perals L. Arachnoid cyst. J Neurosurg. 1996;85:734-5.

13. Van Heerden J, McAuliffe W. Spinal arachnoiditis as a consequence of aneurysm-related subarchnoid haemorrhage. J Med Imaging Radiat Oncol. 2013;57:61-4.

14. Klekamp J. Treatment of syringomyelia related to nontraumatic arachnoid pathologies of the spinal canal. Neurosurgery. 2013;72:376-89.

15. Nakanishi K, Uchiyama T, Nakano N, Fukawa N, Yamada K, Yabuuchi T. Spinal syringomyelia following subarachnoid haemorrhage. J Clin Neurosci. 2012;19:594-7.

16. Sajanti J, Majamaa K. Rapid induction of meningeal collagen synthesis in the cerebral cisternal and ventricular compartments after subarachnoid haemorrhage. Acta Neurochir. 2001;143:821-6.

17. Ovali GY, Adam G, Cinar C, Bozkaya H, Calli C, Kitis O. Symptomatic spinal migration of subarachnoid haemorrhage due to ruptured intradural vertebral artery aneurysm. J Neuroimag. 2015;25:668-70.

18. Chiang Y-C, Lee C-H, Chen W-H, Tsuei Y-S. Acute paraplegia after aneurysmal subarachnoid haemorrhage: a case report of rare complication and review of literature. World Neurosurg. 2016;88: E11-695.

19. Barbaro NM, Wilson CB, Gutin PH, Edwards MS. Surgical treatment of syringomyelia. Favorable results with syringoperitoneal shunting. J Neurosurg. 1984;61:531-8.

20. Caplan LR, Norohna AB, Amico LL. Syringomyelia and arachnoiditis. J Neurol Neurosurg Psychiatry. 1990;53:106-13.

21. Edgar R, Quail P. Progressive post-traumatic cystic and noncystic myelopathy. Br J Neurosurg. 1994;8:7-22.

22. Sgouros S, Williams B. A critical appraisal of drainage in syringomyelia. J Neurosurg. 1995;82:1-10.

23. Klekamp J, Batzdore U, Samii M, Bothe HW. Treatment of syringomyelia associated with arachnoid scarring caused by arachnoiditis or trauma. J Neurosurg. 1997;86:233-40.

24. Morisako H, Takami T, Yamagata T, Chokyu I, Tsuyuguchi N, Ohata K. Focal adhesive arachnoiditis of spinal cord: imaging diagnosis and surgical resolution. J Craniovertebral Junction Spine. 2010;1:100-6.

25. Takami T, Ohata K, Goto T, Nishikawa M, Nishio A, Tsuyuguchi $\mathrm{N}$, et al. Lift-up Laminoplasty for myelopathy caused by ossification of the posterior longitudinal ligament of the cervical spine. Neurol India. 2004;52:59-63.

26. Goto T, Ohata K, Takami T, Nishikawa M, Tsuyuguchi N, Morino M, et al. Hydroxyapatite laminar spacers and titanium miniplates in cervical laminoplasty. J Neurosurg. 2002;97:323-9.

27. Tachibana T, Moriyama T, Maruo K. Subarachnoid-subarachnoid bypass for spinal adhesive arachnoiditis. Case report. J Neurosurg Spine. 2014;21:817-20.

28. Kashcheev AA, Arestov SO, Gushcha AO. Flexible endoscopy in surgical treatment of spinal adhesive arachnoiditis and arachnoid cyst. N N Burdenko J Neurosurg. 2013;5:41-50. 